\title{
Surgery Research and Practice
}

\section{Gram Stain Microscopy in Septic Arthritis}

\section{Soufiane Aharram ${ }^{1 *}$ (D), Jawad Amghar ${ }^{1}$, Mounir Yahyaoui ${ }^{1}$, Omar Agoumi ${ }^{2}$ and Abdelkarim Daoudi ${ }^{3}$}

\author{
${ }^{1}$ Faculty of Medicine and Pharmacy, Department of Traumatology Orthopeadic Surgery, Centre \\ Hospitalier Universitaire Mohammed VI, Morocco \\ ${ }^{2}$ Associate Professor of Orthopaedic and Trauma Surgery, Department of Traumatology Orthopeadic \\ Surgery, Centre Hospitalier Universitaire Mohammed VI, Morocco \\ ${ }^{3}$ Professor of Higher Education in Orthopaedic Surgery and Traumatology, Department of Traumatology \\ Orthopeadic Surgery, Centre Hospitalier Universitaire Mohammed VI, Morocco
}

*Corresponding author: Dr. Soufiane Aharram, Faculty of Medicine and Pharmacy, Department of Traumatology Orthopeadic Surgery, Centre Hospitalier Universitaire VI, Oujda, Morocco, Tel: +212634865947

\begin{abstract}
Background and aim: Septic arthritis is a serious condition that can lead to rapid destruction of joint if it is not rapidly diagnosed and treated appropriately. The reported annual incidence is 10 in 100,000 although this increases to 70 in 100,000 in those with risk factors for developing septic arthritis mainly rheumatoid arthritis and immune-compromised patients. The aim of this study is to examine the sensitivity and specificity, and thus the clinical usefulness, of gram stain results.
\end{abstract}

Materials and methods: Single centre, retrospective case series. All joint aspirates over a three year period from May 2015 to April 2018 were reviewed. The gram stain and final culture results noted.

Results: 830 samples were included from both native and replaced joints. Native joints accounted for a total of 701 cases $(84 \%)$ of the aspirates, whilst those obtained from prosthetic joints $129(16 \%)$. In $74(9 \%)$ cases there was a discrepancy between the gram stain and culture results.

Conclusion: The sensitivity of the gram stain in this case series is $22 \%$ and the specificity of the test is $99.6 \%$. The clinician should have a low threshold for overlooking a negative gram stain result and place greater emphases on the clinical findings in conjunction with biochemical markers.

\section{Keywords}

Septic arthritis, Gram stain, Joint aspirate, Prosthetic joint, Sensitivity

\section{Introduction}

Septic arthritis is a serious condition that can lead to rapid destruction of joint if it is not rapidly diagnosed and treated appropriately. The annual incidence in the US is reported at 10 in 100,000 although this increases to 70 in 100,000 in those with risk factors for developing septic arthritis mainly rheumatoid arthritis and immune-compromised patients. There is also an increased risk in those patients with a prosthetic joint, albeit with a differing consequences [1]. The most common joint affected is the knee, accounting for around $50 \%$ of the cases of septic arthritis, with the hip joint next most involved [2].

Diagnosis can be quite challenging and is usually reached through a combination of assessment of the clinical symptoms and signs, biochemistry, and analysis of an aspirate from the joint. The clinical features are elicited expediently at time of presentation, with biochemistry usually available within hours. Microbiological culture of the joint aspirate often takes many days but damage to the cartilage in the joint happens quickly therefore many clinicians will initiate treatment of the septic arthritis, either with antibiotics, surgical drainage or a combination of the two, prior to the results of the culture being available [3]. A Gram stain of the joint aspirate gives a much quicker result, often within hours, with many clinicians initiating or delaying treatment 
based on that result. However, reported sensitivity of a Gram stain in the literature is low, which puts into question the usefulness of this test.

Cunningham, et al. looked at whether the results of all samples taken in their study (overall sensitivity 0.37 ) varied in specific sub-groups. They found that when they looked at prosthetic infections, concomitant antibiotic therapy, and immunosuppressed patients, the sensitivities were much the same, $0.33,0.36,0.40$ respectively. There was an increased sensitivity, 0.52 , in those with concomitant synovial crystals. All groups had a specificity value $>0.96$ [4]. A study looking at a paediatric population reported sensitivity and specificity values for the detection of septic arthritis of 0.40 and 0.97 respectively, which is comparable to the adult studies [5].

Horowiz, et al. recognised poor sensitivity levels which have been seen in the other studies, and they recommend that synovial fluids should be sent for a combination of a white blood cell count, crystal analysis, Gram stain, and culture; with polymerase chain reaction (PCR) testing to identify less common organisms. They do however acknowledge that the evidence behind such recommendations is of a low level [6]. The evidence does however point more favourably to the use of synovial fluid white blood cell (WBC) counts and percentage of polymorphs in the aspirated joint fluid. A systematic review has shown likelihood ratios increase with WBC counts and polymorphonuclear cell counts; they established a likelihood ratio of 28.0 with WBC counts $>100,000 / \mu \mathrm{L}$, and a likelihood ratio of 3.4 with polymorphonuclear cell counts of at least $90 \%$ compared to 0.34 with counts of less than $90 \%$ [7]. Butler, et al. found that in their series of immunocompromised patients with septic arthritis the average synovial WBC count was 74,190 with a polymorphonuclear cell count of $88 \%$ [8]. These examinations should also be used in conjunction with serum WBCs and CRP measurements, and clinical examinations which together show a strong correlation with the diagnosis of septic arthritis [9].

In this single centre study, our aim was to examine the sensitivity and specificity, and thus the clinical usefulness, of Gram stain results and look at the difference, if any, for the analysis of samples taken from a native and prosthetic joint.

We undertook this research because we believe there are two problems. The first, and more pertinent issue, is the risk of under treating guided by a false negative Gram stain result, which can have catastrophic consequence. The second, although far less common, is potential over treating as the Gram stain returns a positive outcome and the wait for cultures is considered possibly unsafe in certain cases. In many cases, based on the examination and blood markers it can be evident early on that a diagnosis of septic arthritis is very likely and treatment initiated quickly. In other cases, diag- nosis can be difficult and therefore greater emphasis is placed on the Gram stain result.

\section{Materials and Methods}

This was a retrospective single center study (CHU Mohammed VI, Oujda, Morocco). All joint aspirates over a three year period from May 2015 to April 2018 performed at our hospital were reviewed. This was provided by the microbiology department and included aspirates from the emergency department, all wards and both orthopaedic and rheumatology outpatients. Aspirates were excluded if no patient number had been recorded or the aspirate type and source were not available, as accurate results could not be obtained. We also excluded non joint aspirates such as ascitic or pleural samples.

All aspirates were reviewed, irrespective of point of the patient presentation or the indication for aspiration. We included both native and prosthetic joints. If a patient had had multiple joint aspirations over this time period each aspirate was analysed individually and included.

Each aspirate had been sent to the microbiology laboratory for a Gram stain and microbiological culture. Some samples were also sent for PCR where there was a high index of suspicion on infection with a negative microbiological culture. For each sample the result of the initial Gram stain and the final cultures were noted. For each aspirate the joint aspirated was also recorded. The microbiology department has two trained personnel who examine the Gram stain "in-house". The data was analysed by the lead author.

\section{Results}

The search generated a total of 830 eligible joint aspirates which were recorded and analysed by the microbiology department over the defined period. Native joints accounted for a total of 698 cases (84\%) of the aspirates, whilst those obtained from prosthetic joints $132(16 \%)$. The majority of samples were from the knee joint ( $n=612,74 \%$ ) with the remainder from the hip ( $n$ $=57)$, shoulder $(n=45)$, elbow $(n=48)$, wrist $(n=35)$, and ankle $(n=28)$. There were also few samples from the small joints of hand and feet.

There was a match between the Gram stain and culture result in 756 cases (91\%), within which were only 20 cases where both the Gram stain and the culture were both positive.

In 74 (9\%) cases there was a discrepancy between Gram stain and culture results. The majority, 71 of the samples, had a negative Gram stain assessment but positive culture growth; either through normal culture, prolonged culture or PCR. For 3 of the samples, a positive Gram stain was recorded with a final negative culture results. 
Samples from prosthetic joints accounted for 31\% of cases where there was Gram stain and culture mismatch, with over representation as percentage of samples of prosthetic joints in the study in total was $16 \%$.

In a subgroup analysis, looking at sensitivity and specificity for native and prosthetic joints separately, the results were mostly comparable, with slight better sensitivity in the prosthetic cohort. In the prosthetic group the sensitivity was $23 \%$, whilst for the native group it was $21 \%$. The specificity was $100 \%$ and $99.5 \%$ respectively.

\section{Discussion}

In this study we found that the sensitivity of a positive Gram stain was very low at $22 \%$, lower than what has previously been reported in the literature where the range has been quoted from $29 \%$ to $60 \%[4,7]$. This is problematic as a large proportion of cases of septic arthritis will not be detected by this initial Gram stain. Therefore the Gram stain is of questionable value when assessing patients for septic arthritis as it is not able to identify those patients which truly have the disease. However, we found the specificity to be nearly $100 \%$ $(99.6 \%)$ for the test. It is therefore a good tool to tell us which patients do not have the disease but unable to reliably pick up those who do have the disease.

With the test of such low diagnostic sensitivity we would question why a Gram stain is used as part of the normal investigations when septic arthritis is suspected. Clinical findings such as joint pain, joint swelling and fever have all had a reported higher sensitivity of $85 \%$, $78 \%$ and $57 \%$ for each domain [7]. Blood test including a high neutrophil proportion (of over 90\%) and CRP have also be shown to be predictive of septic arthritis $[7,10]$. Therefore we carefully consider Gram stain use and place greater emphasis to manage the patient based on clinical presentation and biochemical findings. The joint will still require aspiration so that fluid can be sent for culture to obtain a diagnosis is and guide antibiotic therapy. If clinically suspected treatment should not be delayed until the availability of a Gram stain result.

We were unable to ascertain whether antibiotic therapy had been initiated or not prior to obtaining the sample. This can alter the result of both the Gram stain and culture results. Although there are two trained personnel who had analysed the Gram stain result, we did not have a record of which one of them analysed which sample. As the Gram stain is operator dependent this can be a confounding factor. We consider these as limitations to our study.

With regards to prosthetic joints making up a higher sample representation in those results where there is a mismatch, perhaps this is amplified as prosthetic infection often necessitate for multiple samples to be sent and analysed. In our study we initially processed each sample as an individual sample even if it were taken from the same joint at the same time, however, we did look further into this, and accounting for duplicate samples (10 samples were identified) the prosthetic joints account for $15 \%$ of the total samples and $27 \%$ of the samples that showed a mismatch. We also know that these may be chronic, and may have had previous antibiotic treatment. The biology may also change and biofilm can form which again may affect Gram stain results. Further research into this area is required to study this aspect in particular.

In this study, we had larger numbers compared to previous published series, specifically looked at both native and prosthetic cohorts, and had a lower overall sensitivity than previously reported.

In conclusion, the diagnosis of septic arthritis can sometimes be complex and difficult. Gram stain tests have low sensitivity and therefore the clinician should have a low threshold for overlooking a negative Gram stain result and place greater emphases on the clinical findings and biochemical and haematological markers.

\section{Funding}

This research did not receive any specific grant from funding agencies in the public, commercial, or not-forprofit sectors.

\section{Conflict of Interest}

No conflict of interest.

\section{Ethical Approval}

This article does not contain any studies conducted by either author on human or animal participants.

\section{Informed Consent}

Informed consent was obtained from all individual participants included in the study.

\section{References}

1. Kaandorp CJ, Van Schaardenburg D, Krijnen P, Habbema JD, Van De Laar MA (1995) Risk factors for septic arthritis in patients with joint disease. A prospective study. Arthritis Rheum 38: 1819-1825.

2. Kaandorp CJ, Dinant HJ, van De Laar MA, Moens HJ, Prins AP, et al. (1997) Incidence and sources of native and prosthetic joint infection: A community based prospective survey. Ann Rheum Dis 56: 470-475.

3. Siva C, Velazquez C, Mody A, Brasington R (2003) Diagnosing acute monoarthritis in adults: A practical approach for the family physician. Am Fam Physician 68: 83-90.

4. Cunningham G, Seghrouchni K, Ruffieux E, Vaudaux $P$, Gayet-Ageron A, et al. (2014) Gram and acridine orange staining for diagnosis of septic arthritis in different patient populations. Int Orthop 38: 1283-1290.

5. Bram JT, Baldwin KD, Blumberg TJ (2018) Gram stain is not clinically relevant in treatment of pediatric septic arthritis. J Pediatr Orthop 38: 536-540.

6. Horowitz DL, Katzap E, Horowitz S, Barilla-LaBarca ML (2011) Approach to septic arthritis. Am Fam Physician 84: 653-660. 
7. Margaretten ME, Kohlwes J, Moore D, Bent S (2007) Does this adult patient have septic arthritis? JAMA 297: 14781488.

8. Butler BA, Fitz DW, Lawton CD, Li DD, Balderama ES, et al. (2018) Early diagnosis of septic arthritis in immunocompromised patients. J Orthop Sci 23: 542-545.
9. Faraj AA, Omonbude OD, Godwin P (2002) Gram staining in the diagnosis of acute septic arthritis. Acta Orthop Belg 68: 388-391.

10. Ernst AA, Weiss SJ, Tracy LA, Weiss NR (2010) Usefulness of CRP and ESR in predicting septic joints. South Med J 103: 522-526. 